\title{
Retracción química de pastas de cemento con incorporación de metacaolín
}

\author{
Chemical shrinkage of cementitious pastes with \\ metakaulin incorporation
}

\begin{tabular}{|c|c|}
\hline & $\begin{array}{l}\text { Delver Plinio Estrada Caceres } \\
\text { Roberto Cesar de Olivera Romano } \\
\text { Vanderley Moacyr J ohn } \\
\text { Maria Alba Cincotto }\end{array}$ \\
\hline Iver Plinio Estrada Caceres & $\begin{array}{l}\text { Resumen } \\
\text { ste estudio muestra la influencia que produce la sustitución parcial de } \\
\text { cemento Portland por metacaolín, en las reacciones de hidratación y } \\
\text { retracción química de pastas cementicias. Para tal fin se elaboraron } \\
\text { pastas utilizando reemplazos de metacaolín de } 5,10 \text { y } 20 \% \text { en masa, } \\
\text { además de una pasta de referencia, usando una relación constante agua/sólido de } \\
\text { 0,5. La retracción química fue medida en forma continua automatizando el método } \\
\text { de la dilatometría, también se evaluó la cinética de hidratación usando un } \\
\text { calorímetro isotérmico. Adicionalmente, el desarrollo de la microestructura fue } \\
\text { estudiado por análisis termogravimétrico y difracción de rayos X. Los resultados } \\
\text { obtenidos muestran que existe una excelente relación entre la retracción química y } \\
\text { la calorimetría y que la adición de metacaolín produce cambios en la cinética de } \\
\text { hidratación, que también afectan a la retracción química. }\end{array}$ \\
\hline $\begin{array}{l}\text { Universidade de São Paulo } \\
\text { São Paulo - SP - Brasil }\end{array}$ & $\begin{array}{l}\text { Palabras-clave: Retracción química. Metacaolín. Cemento Portland. Cinética } \\
\text { de hidratación. }\end{array}$ \\
\hline $\begin{array}{r}\text { Roberto Cesar de Oliveira } \\
\text { Romano }\end{array}$ & Abstract \\
\hline $\begin{array}{l}\text { Universidade de São Paulo } \\
\text { São Paulo - SP - Brasil }\end{array}$ & $\begin{array}{l}\text { This study shows the influence of partial replacement of Portland cement by } \\
\text { metakaolin in the hydration reactions and chemical shrinkage of cementitious }\end{array}$ \\
\hline $\begin{array}{l}\text { Vanderley Moacyr J ohn } \\
\text { Universidade de São Paulo } \\
\text { São Paulo - SP - Brasil }\end{array}$ & $\begin{array}{l}\text { pastes. For this, pastes were prepared using metakaolin replacements of 5, IU and } \\
\text { Chemical shrinkage was measured continuously automating the method of } \\
\text { dilatometry, hydration kinetics was evaluated using an isothermal conduction }\end{array}$ \\
\hline $\begin{array}{l}\text { Maria Alba Cincotto } \\
\text { Universidade de São Paulo } \\
\text { São Paulo - SP - Brasil }\end{array}$ & $\begin{array}{l}\text { calorimetry. Additionally, the development of the microstructure was studied by } \\
\text { thermogravimetric analysis and X-ray diffraction. The results show that there is an } \\
\text { excellent relationship between chemical shrinkage and calorimetry and the } \\
\text { addition of metakaolin leads to changes of the hydration kinetics, which also affect } \\
\text { the chemical shrinkage. }\end{array}$ \\
\hline & : Chemical shrinkage. Metakaolin. Portland cement. Kinetics of hydration. \\
\hline
\end{tabular}

CACERES, D. P. E.; ROMANO, R. C. de O.; J OHN, V. M.; CINCOTTO, M. A. Retracción química de pastas de cemento 


\section{Introducción}

La utilización de metacaolín (MK) como material cementicio suplementario (SCM) es una práctica común en el sector de la construcción, debido a que permite la reducción de la cantidad de cemento en las composiciones.

Su calidad y desempeño dependen de factores como: propiedades geológicas y mineralógicas de su materia prima (caolín) y la eficiencia en el tratamiento térmico (transformación de caolín a metacaolín). El MK está conformado principalmente por sílice $\left(\mathrm{SiO}_{2}, 50\right.$ a 55\%) y alúmina $\left(\mathrm{Al}_{2} \mathrm{O}_{3}, 40\right.$ a 45\%) (POON et al., 2001), también puede contener $\mathrm{Fe}_{2} \mathrm{O}_{3}, \mathrm{TiO}_{2}, \mathrm{CaO}, \mathrm{MgO}$, entre otros contaminantes (SABIR; WILD; BAI, 2001; SIDDIQUE; KLAUS, 2009). Tiene como características principales: morfología plana, tamaño de partículas menores a $20 \mu \mathrm{m}$ (AMBROISE; MAXIMILIEN; PERA, 1994), área superficial específica que varía entre 10 a $29 \mathrm{~m}^{2} / \mathrm{g}$, su tonalidad varía desde blanca hasta rosada (RASHAD, 2013), dependiendo de la proporción de las especies químicas que lo componen.

La inclusión de MK en las composiciones cementicias altera la cinética y reacciones de hidratación, el desarrollo de la microestructura y las propiedades en estado fresco del cemento Portland. Esto es debido a que la adición de MK en pastas cementicias, resulta en efecto filler durante las primeras edades, aumentando la cantidad de puntos de nucleación, favoreciendo así el desarrollo de la hidratación del $\mathrm{C}_{3} \mathrm{~S}$ del cemento Portland. Por su parte, la reacción puzolánica ocurre en edades avanzadas, generalmente mayores a 24 horas dependiendo de su reactividad (LOTHENBACH; SCRIVENER; HOOTON, 2011; SCRIVENER; JUILLAND; MONTEIRO, 2015; BERODIER, 2015). También, es alterada la retracción química, la cual es definida como la disminución de volumen absoluto, debido a que los productos hidratados ocupan menos volumen que los reactantes (agua, cemento, SCM). Asimismo, está directamente relacionada con el grado de hidratación y el calor liberado de las pastas (PARROTT et al., 1990).

Normalmente para evaluar la cinética de hidratación se analiza el calor liberado, usando un calorímetro de conducción isotérmico. Sin embargo, otra técnica relativamente nueva para este propósito, es medir la retracción química en forma continua, que se consigue automatizando su adquisición de datos. En Brasil son raros los estudios que usen retracción química para obtener la cinética de hidratación. Determinar la retracción química en comparación a la calorimetría tiene como ventajas: ser más económica y de fácil implementación, además de posibilitar ensayos de larga duración.

Anteriormente fue realizado un levantamiento bibliográfico de los métodos que existen para determinar la retracción química (ESTRADA; JOHN; CINCOTTO, 2017). Teniendo en cuenta este trabajo, en el presente estudio se usó y automatizó el método de dilatometría especificado en la norma C-1608 (AMERICAN..., 2012), con el objetivo de determinar la influencia que tiene la utilización del MK en la retracción química y reacciones de hidratación de mezclas con cemento Portland.

\section{Materiales y métodos}

Se utilizó cemento Portland de alta resistencia inicial CPV y metacaolín, los cuales presentan distribución del tamaño de partículas (determinado por difracción de rayos laser) presentada en la Figura 1a, microscopia electrónica de barredura (MEB) mostrado en la Figura 1b y 1c; área superficial específica (BET), densidad, índice de actividad puzolánica (Chapelle modificado) y diámetros $\left(\mathrm{d}_{10}, \mathrm{~d}_{50}\right.$ y $\left.\mathrm{d}_{90}\right)$ mostrados en la Tabla 1 ; composición química, determinada por fluorescencia de rayos $\mathrm{X}$, conforme se presenta en la Tabla 2.

El MK presenta densidad y área superficial específica, notablemente distintas en comparación al cemento Portland. De esta forma, se puede decir que existe un considerable impacto en la movilidad de las partículas de la pasta: cuanto mayor es la cantidad de sustitución, menor es la distancia de separación entre las partículas, afectando el flujo. Este hecho resulta en alteraciones en las propiedades reológicas y necesidad de aumento de la demanda de agua para el mezclado, siempre y cuando se opte por mantener la consistencia de las composiciones. Sin embargo, como el objetivo de este trabajo es evaluar solamente el impacto del uso del MK en la retracción química y reacciones de hidratación del cemento, se optó por eliminar las posibles variables como: cantidad de agua, temperatura de ensayo, forma de mezclado de la pasta.

A pesar de ser típicamente indicado que el tamaño de partículas del MK es más fina que la del cemento Portland, el MK utilizado en este trabajo no presenta tal característica, teniendo mayor cantidad de partículas más gruesas que las del cemento, como se observa en $d_{50}$ y $d_{90}$ (Tabla 1), desplazando ligeramente su curva de distribución granulométrica hacia la derecha (Figura 1a). 
Figura 1 - (a) Distribución granulométrica de los materiales, (b) y (c) MEB del MK
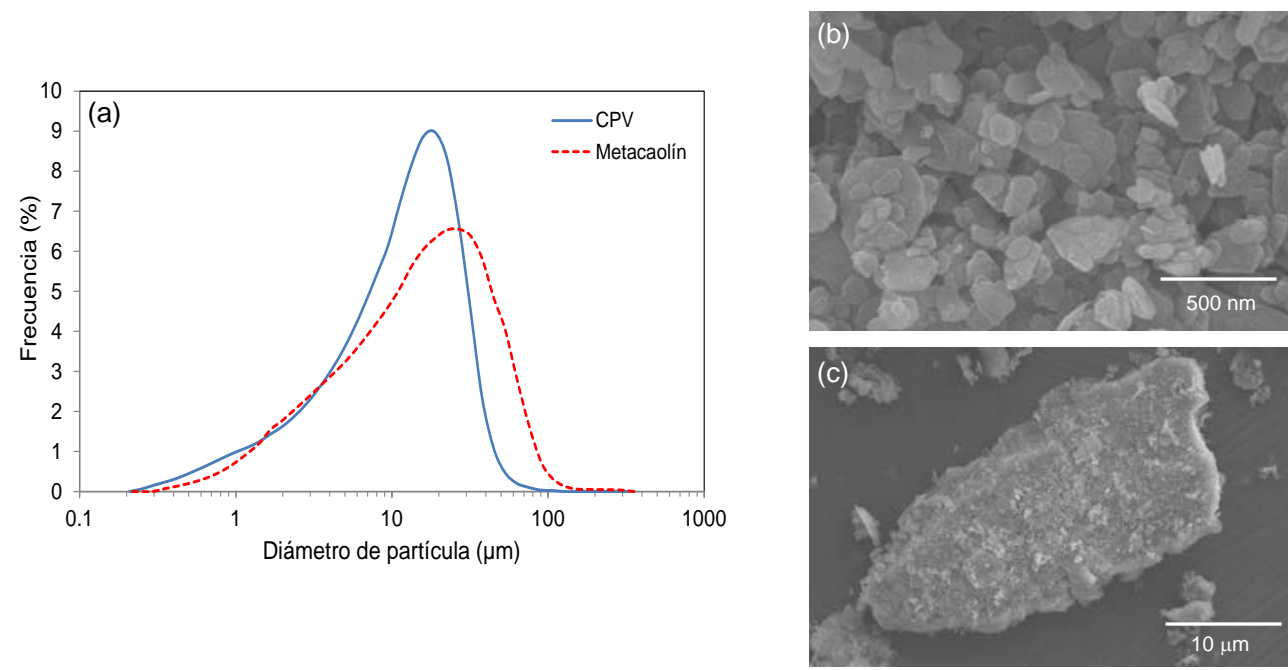

Tabla 1 - Características físicas de los materiales

\begin{tabular}{l|c|c}
\hline \multicolumn{1}{c|}{ Características } & Cemento & Metacaolín \\
\hline Área superficial específica BET $\left(\mathrm{m}^{2} / \mathrm{g}\right)$ & $1,2 \pm 0,02$ & $17,2 \pm 0,02$ \\
Densidad $\left(\mathrm{g} / \mathrm{cm}^{3}\right)$ & $3,0 \pm 0,01$ & $2,6 \pm 0,04$ \\
Índice de actividad puzolánica $\left[\mathrm{mg} \mathrm{Ca}(\mathrm{OH})_{2} / \mathrm{g}\right]$ & - & $1106 \pm 0,01$ \\
$\mathrm{~d}_{10}(\mu \mathrm{m})$ & 2 & 2,8 \\
$\mathrm{~d}_{50}(\mu \mathrm{m})$ & 15 & 15 \\
$\mathrm{~d}_{90}(\mu \mathrm{m})$ & 34 & 55 \\
\hline
\end{tabular}

Tabla 2 - Composición química de los materiales (\%)

\begin{tabular}{l|c|c|c|c|c|c|c|c|c|c|c|c|c|c}
\hline Material & $\mathbf{C a O}$ & $\mathbf{S i O}_{2}$ & $\begin{array}{c}\mathbf{A l}_{2} \mathbf{O} \\
\mathbf{3}\end{array}$ & $\mathbf{F e}_{2} \mathbf{O}_{3}$ & $\begin{array}{c}\mathbf{M g} \\
\mathbf{O}\end{array}$ & $\mathbf{S O}_{3}$ & $\begin{array}{c}\mathbf{N a}_{2} \\
\mathbf{O}\end{array}$ & $\begin{array}{c}\mathbf{K}_{2} \\
\mathbf{O}\end{array}$ & $\begin{array}{c}\mathbf{T i O} \\
2\end{array}$ & $\mathbf{P}_{2} \mathbf{O}_{5}$ & $\begin{array}{c}\mathbf{M n} \\
\mathbf{O}\end{array}$ & SrO & P.C.* & $\begin{array}{c}\text { Tota } \\
\mathbf{l}\end{array}$ \\
\hline $\mathrm{CPV}$ & 64,5 & 20,0 & 4,3 & 2,5 & 1,4 & 0,9 & 0,2 & 0,8 & 0,2 & 0,2 & 0,11 & 0,2 & 4,4 & 100 \\
$\mathrm{MK}$ & $<0,1$ & 60,9 & 30,3 & 1,7 & 0,5 & - & $<0,1$ & 1,3 & 1,7 & $<0,1$ & 0,02 & - & 3,5 & 100 \\
\hline
\end{tabular}

Nota: *P.C. - Pérdida por calcinación.

El cemento Portland escogido para la realización de este trabajo es del tipo más puro comercializado en Brasil, con elevada cantidad de clinker, adición de calcáreo de 5\% como máximo y sulfato de calcio menor que $3 \%$.

Este hecho puede ser explicado debido a la morfología de las partículas del MK, la que fue analizada por MEB, usando un equipo Quanta 600FEG. En ella se observa que el MK tiene una morfología laminar (Figura 1b) y que al analizar su granulometría por luz láser, hace que se considere un diámetro mayor que depende de su orientación (Figura 1c). Otra consideración es que, durante el ensayo el MK puede estar aglomerado debido a su elevada área superficial específica. No obstante, esto no compromete los resultados obtenidos, puesto que el trabajo trata de una evaluación comparativa entre los contenidos de MK.
En la Figura 2a se observa los difractogramas correspondientes a los materiales anhidros. Para el caso del MK el pico de mayor intensidad corresponde al cuarzo y moscovita localizado entre $26^{\circ}$ a $27^{\circ} 2 \theta$, también se observan varios picos correspondientes a la caolinita, esto indica que el material no fue debidamente calcinado. Mientras que, el cemento Portland presenta un difractograma típico del ligante más puro comercializado en Brasil. Asimismo, en la Tabla 3, se indican las nomenclaturas, fórmulas moleculares $\mathrm{y}$ fichas consultadas de las fases mineralógicas encontradas en los difractogramas.

La Figura 2b muestra la pérdida de masa de las materias primas en función de la variación de la temperatura. A pesar de no haber sido identificado en el difractograma, fue posible observar que existe $3,1 \%$ de portlandita en el cemento, indicando una pequeña pre-hidratación y 5.6\% de carbonato de calcio debido a la adición utilizada en el ligante. 
Para calcular cuánto de caolín no fue debidamente calcinado, se usa la relación $\mathrm{Al}_{2} \mathrm{O}_{3} .2 \mathrm{SiO}_{2} .2 \mathrm{H}_{2} \mathrm{O} / 2 \mathrm{H}_{2} \mathrm{O}$ multiplicada por la cantidad de agua de deshidroxilación (pérdida de masa entre 400 a $600{ }^{\circ} \mathrm{C}$ ), esto es $258 / 36$ x 1,73 , resultando $12,4 \%$ que corresponde al caolín remaneciente, el resto $87.6 \%$ es $\mathrm{MK}$ y minerales accesorios.

Las composiciones de las pastas evaluadas se resumen en la Tabla 4. La primera únicamente utilizando cemento Portland como referencia y las otras con incrementos de 5, 10 y 20\% de sustitución parcial de MK en masa.

Todas las pastas fueron elaboradas usando una relación agua/sólido de 0,5 y se prepararon de la siguiente forma: primero, se añadió agua a los materiales sólidos y se dejó en reposo durante 30 segundos, en seguida, se realizó el mezclado durante 90 segundos, usando un mezclador de alta energía (Makita, adaptado), controlando la velocidad de giro en $10000 \mathrm{rpm}$. Cabe mencionar que cuando se usaron sustituciones de 5, 10 y 20\% de metacaolín, los materiales anhidros fueron homogenizados previamente usando un mezclador (Makita, Labortechnik RW 20D2M) con una velocidad de giro de $1000 \mathrm{rpm}$. Es necesario resaltar que ésta es una etapa muy importante en el proceso experimental, ya que la calidad de la mezcla puede cambiar la reacción del cemento.

Figura 2 - (a) Difractogramas de rayos $X$ de los materiales anhidros - K: caolinita, Q: cuarzo, M: moscovita, $A: C_{3} S, B: C_{2} S, A C$ : $C_{3} A$, G: gipsita, Ca: calcita y Br: brownmillerita; (b) Curvas termogravimétricas

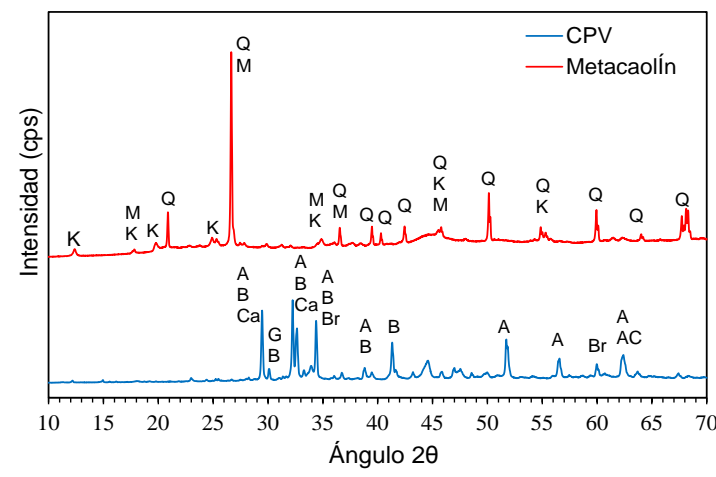

(a)

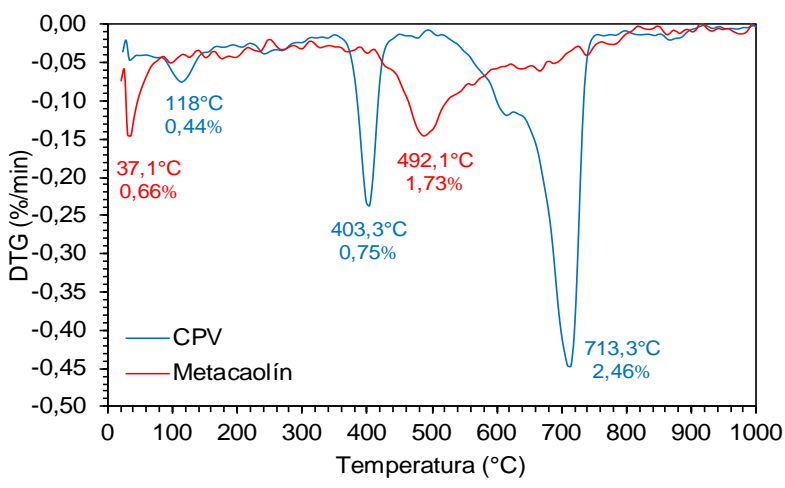

(b)

Tabla 3 - Fases mineralógicas

\begin{tabular}{c|l|l|c}
\hline Nomenclatura & \multicolumn{1}{|c|}{ Fase mineralógica } & \multicolumn{1}{c}{ Fórmula molecular } & Ficha consultada \\
\hline $\mathrm{A}$ & Alita $-\mathrm{C}_{3} \mathrm{~S}$ & $3 \mathrm{CaO} \cdot \mathrm{SiO}_{2}$ & $\mathrm{ICOD} 00-042-0551$ \\
$\mathrm{~B}$ & Belita $-\mathrm{C}_{2} \mathrm{~S}$ & $2 \mathrm{CaO} \cdot \mathrm{SiO}_{2}$ & $\mathrm{ICOD} 01-073-2091$ \\
$\mathrm{AC}$ & Aluminato tricálcico $-\mathrm{C}_{3} \mathrm{~A}$ & $3 \mathrm{CaO} \cdot \mathrm{Al}_{2} \mathrm{O}_{3}$ & $\mathrm{ICOD} 00-001-1060$ \\
$\mathrm{Br}$ & Brownmilerita $-\mathrm{C} 4 \mathrm{AF}$ & $4 \mathrm{CaO} \cdot \mathrm{Al}_{2} \mathrm{O}_{3} \cdot \mathrm{Fe}_{2} \mathrm{O}_{3}$ & $\mathrm{COD} 96-900-3349$ \\
$\mathrm{Ca}$ & Calcita & $\mathrm{CaCO}_{3}$ & $\mathrm{ICOD} 01-072-1651$ \\
$\mathrm{G}$ & Gipsita & $\mathrm{CaSO}_{4} \cdot 2 \mathrm{H}_{2} \mathrm{O}$ & $\mathrm{COD} 96-101-0982$ \\
$\mathrm{Q}$ & Cuarzo & $\mathrm{SiO}_{2}$ & $\mathrm{COD} 96-900-9667$ \\
$\mathrm{~K}$ & Caolinita & $\mathrm{Al}_{2} \mathrm{Si}_{2} \mathrm{O}_{5}(\mathrm{OH})_{4}$ & $\mathrm{COD} 96-900-9235$ \\
$\mathrm{M}$ & Muscovita & $\mathrm{KAl}_{2}\left(\mathrm{AlSi}_{3} \mathrm{O}_{10}\right)(\mathrm{F}, \mathrm{OH})_{2}$ & $\mathrm{COD} 96-900-5188$ \\
\hline
\end{tabular}

Tabla 4 - Composiciones evaluadas

\begin{tabular}{l|c|c}
\hline \multirow{2}{*}{ Denominación } & \multicolumn{2}{|c}{ Composición en masa (\%) } \\
\cline { 2 - 3 } & Cemento & Metacaolín \\
\hline CPV 0\%MK & 100 & 0 \\
CPV 5\%MK & 95 & 5 \\
CPV 10\%MK & 90 & 10 \\
CPV 20\%MK & 80 & 20 \\
\hline
\end{tabular}


Para el estudio del flujo de calor, se usó un calorímetro de conducción isotérmico de la marca TAM Air Thermometric. Las pastas se colocaron dentro de frascos de vidrio cilíndricos, con diámetro de 22,5 mm y altura de $47 \mathrm{~mm}$, enseguida, fueron tapados y ubicados en las celdas del calorímetro. El flujo de calor fue medido durante 72 horas. La temperatura fue mantenida en $25{ }^{\circ} \mathrm{C}$ durante el ensayo.

La Figura 3 muestra detalles de la adaptación del método para medir la retracción química en forma continua, para lo cual se automatizó el método de dilatometría que se especifica en la norma C-1608 (AMERICAN..., 2012). Se colocaron aproximadamente $14 \mathrm{~g}$ de pasta de cemento, mezclados como ya fue descrito anteriormente, en frascos de vidrio iguales a los utilizados en los ensayos de calorimetría, con espesor de pasta de 20 $\mathrm{mm}$. La superficie de la pasta fue cubierta con una delgada capa de agua desionizada de 2 g. Luego, el resto del frasco fue rellenado con aceite de parafina líquida teñida de color rojo para facilitar la lectura. Después el frasco fue sellado con una tapa de hule que tiene un orificio en su parte central, en el cual está insertada una pipeta con capacidad de $2 \mathrm{~mL}$, graduada a $0,01 \mathrm{~mL}$. Con la ayuda de una jeringa se rellenó parafina líquida hasta completar el nivel inicial de lectura deseado. Por último, los frascos fueron colocados en un baño térmico con temperatura controlada a $25 \pm 1{ }^{\circ} \mathrm{C}$.

Una vez culminada la etapa experimental descrita en el párrafo anterior, se inició con la adquisición y procesamiento de datos. Las lecturas se realizaron en fotografías, que se obtuvieron usando una cámara web (Logitech, C920) conectada a un computador y controlado por un software de adquisición de imágenes (Chronolapse, versión 1.0.7). Las fotografías se realizaron a intervalos de 1 hora, durante 72 horas y en 3 muestras repetidas. Con respecto al tratamiento de datos, debido a que la curva obtenida presentó oscilaciones sobre todo en edades avanzadas, se procedió a suavizarla usando el software Origin versión 6.0, que también sirvió para obtener la curva derivada. Mayores detalles del procedimiento utilizado, adquisición y procesamiento de datos, así como los cuidados al momento de realizar el ensayo se encuentran en el estudio de Estrada (2016).

\section{Figura 3 - Fotografías de la adaptación del método}

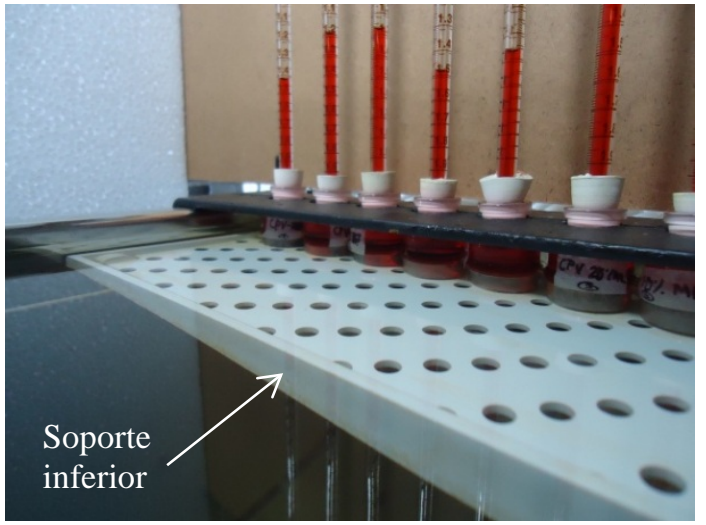

(a) Soporte inferior

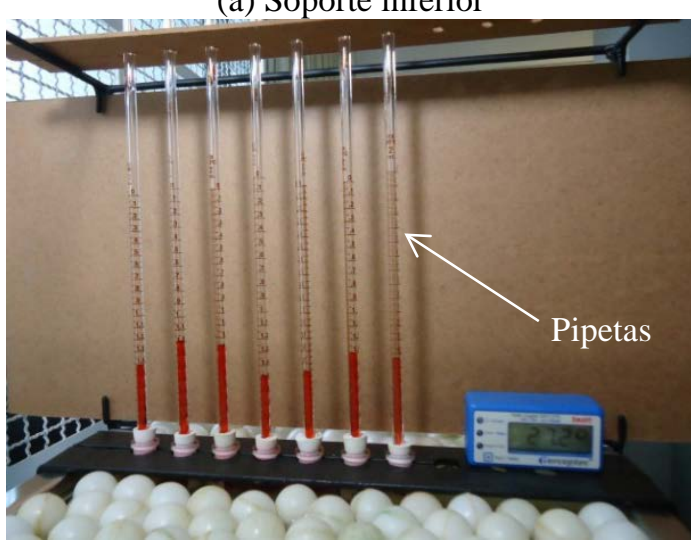

(c) Pipetas

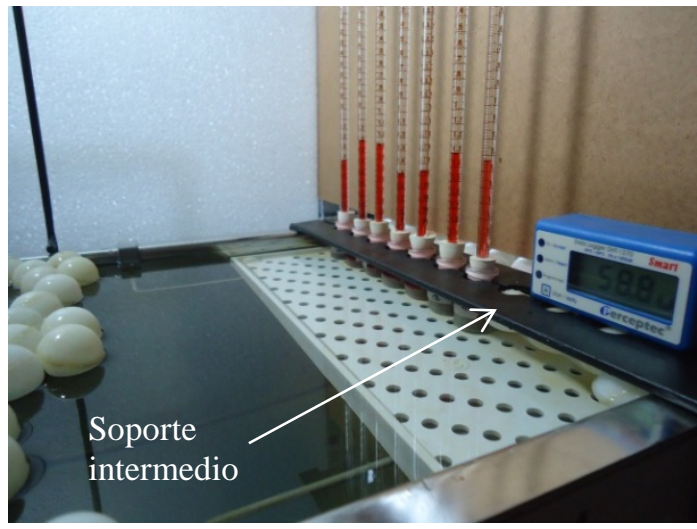

(b) Soporte intermedio

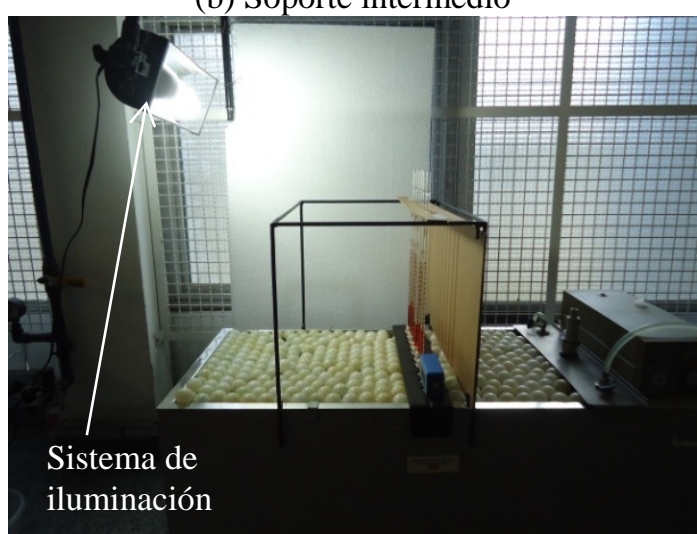

(d) Sistema de iluminación 
También se acompañó la evolución de la cantidad de agua combinada $\left(50\right.$ a $350{ }^{\circ} \mathrm{C}$ ) y de portlandita (400 a $500{ }^{\circ} \mathrm{C}$ ) durante la hidratación, por medio de análisis termogravimétrico, utilizando una termobalanza (Netzsch, TG209 F1). Esto una vez que la hidratación de las pastas fueron paralizadas sumergiéndolas en nitrógeno líquido, posteriormente secadas por liofilización, finalmente molidas y mantenidas en un desecador. Así mismo, las fases cristalinas fueron identificadas mediante difracción de rayos X (DRX), usando un difractómetro (Bruker, D8 Endeavor). Las condiciones de análisis fueron: tubo de $\mathrm{Cu}$, energía $40 \mathrm{~mA}$ x $40 \mathrm{kV}$, ángulo $5-70^{\circ} 2 \theta$, paso angular $0,02^{\circ}$ y 30 s por paso.

\section{Resultados y discusión}

\section{Calorimetría}

La Figura 4a muestra el calor acumulado hasta 72 horas de hidratación, mientras que en la Figura 4b se observa el flujo de calor hasta 36 horas, obviando el tiempo restante para que se aprecien mejor los períodos de aceleración y desaceleración. Esto para la pasta de referencia y sustituciones de 5, 10 y $20 \%$ de MK. Los resultados están normalizados con respecto al contenido de cemento.

En la Figura 4a, se observa que el calor acumulado aumenta con la sustitución gradual de MK. En la Tabla 5, se muestran los valores de calor acumulado. A las 72 horas de hidratación, el cemento Portland sin sustitución de MK generó un calor acumulado de 330,8 J/g, mientras que para
20\% llegó a $407.6 \mathrm{~J} / \mathrm{g}$ (23.2\% de incremento). Este aumento de calor liberado, es debido a que la presencia de MK hace que ocurran reacciones exotérmicas adicionales, debido al efecto filler, que favorece la nucleación del C-S-H, a causa de su elevada área superficial específica y cantidad adicionada (LAGIER; KURTIS, 2007).

En la Figura 4b, se aprecia que existe un punto de inflexión en la etapa de aceleración, indicando el punto de consumo total de sulfato conforme a la norma C-1679 (AMERICAN..., 2014). En cuanto a las modificaciones que ocurren durante el período de aceleración (Tabla 6), su duración disminuye, existe una diferencia de 3 horas entre la pasta de referencia y la mayor sustitución. Asimismo, la tasa de reacción es favorecida, aumentándose el flujo de calor al final del período de aceleración de 5,56 a $8,04 \mathrm{~mW} / \mathrm{g}$. Estas modificaciones indican que la inclusión de MK acelera las reacciones de hidratación durante esta etapa, principalmente formando mayor cantidad de C-S-H, debido a los puntos extras de nucleación (LOTHENBACH; SCRIVENER; HOOTON, 2011; JUENGER; SIDDIQUE, 2015; SCRIVENER; JUILLAND; MONTEIRO, 2015). Estudios recientes mencionan que la inclusión de SCM reduce la distancia entre partículas, esta reducción depende de condiciones de cizallamiento (BERODIER; SCRIVENER, 2014), de este modo, la reacción de los componentes del cemento Portland son favorecidos. Estos resultados confirman los obtenidos anteriormente por Lagier y Kurtis (2007), Antoni et al. (2012) y Vance et al.(2013).

Figura 4 - (a) Calor acumulado hasta 72 horas y (b) Flujo de calor hasta 36 horas - para la pasta de referencia y con sustituciones de 5,10 y $20 \%$ de $M K$
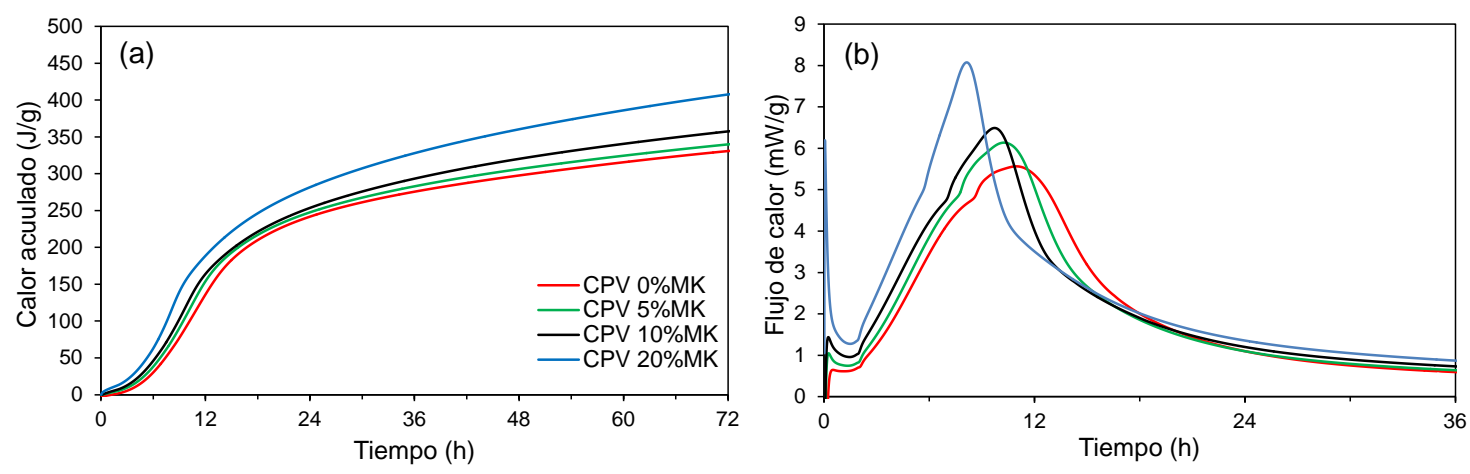

Tabla 5 - Resultados de calor acumulado para sustituciones de 5, 10 y $20 \%$ de MK

\begin{tabular}{c|c|c|c|c}
\hline \multirow{2}{*}{ Muestra } & \multicolumn{4}{|c}{ Calor acumulado (J/g) } \\
\cline { 2 - 5 } & $\mathbf{1 2} \mathbf{~ h}$ & $\mathbf{2 4} \mathbf{~ h}$ & $\mathbf{4 8 ~ h}$ & $\mathbf{7 2} \mathbf{~ h}$ \\
\hline CPV 0\%MK & $136,0(-)$ & $241,7(-)$ & $297,6(-)$ & $330,8(-)$ \\
CPV 5\%MK & $153,7(+13,0 \%)$ & $247,6(+2,4 \%)$ & $306,3(+2,9 \%)$ & $339,8(+2,7 \%)$ \\
CPV 10\%MK & $163,7(+20,4 \%)$ & $253,6(+4,9 \%)$ & $320,2(+7,6 \%)$ & $357,5(+8,1 \%)$ \\
CPV 20\%MK & $188,5(+38,6 \%)$ & $281,6(+16,5 \%)$ & $360,3(+21,1 \%)$ & $407,6(+23,2 \%)$ \\
\hline
\end{tabular}


Tabla 6 - Tiempo de duración, flujo de calor y tiempo de fragua durante el período de aceleración

\begin{tabular}{|c|c|c|c|c|c|c|}
\hline \multicolumn{7}{|c|}{ Período de aceleración } \\
\hline \multirow[t]{2}{*}{ Muestra } & Inicio & Final & $\begin{array}{c}\text { Tiempo } \\
\text { duración }\end{array}$ & $\begin{array}{l}\text { Flujo de } \\
\text { calor }\end{array}$ & $\begin{array}{l}\text { Tasa de } \\
\text { reacción }\end{array}$ & $\begin{array}{l}\text { Tiempo de } \\
\text { fragua* }\end{array}$ \\
\hline & (h) & (h) & (h) & $(\mathrm{mW} / \mathrm{g})$ & (mW/gh) & (h) \\
\hline CPV 0\%MK & 2 & 11 & 9 & 5,56 & 0,72 & 5,5 \\
\hline CPV 5\%MK & 2 & 10 & 8 & 6,12 & 0,82 & 5 \\
\hline CPV 10\%MK & 2 & 9,8 & 7,8 & 6,49 & 0,84 & 4,9 \\
\hline CPV 20\%MK & 2 & 8 & 6 & 8,04 & 0,99 & 4 \\
\hline
\end{tabular}

Nota: *tiempo de fragua, obtenido a partir del 50\%del final del período de aceleración según ASTM C-1679 (2014).

De las curvas de calorimetría y teniendo en cuenta las modificaciones que produce el MK en edades iniciales, desde el punto de vista práctico, con la inclusión cada vez mayor de MK, el tiempo de fragua disminuye de 5,5 a 4 h (Tabla 6), por lo cual el tiempo de trabajabilidad se reduce. Este efecto es debido al aumento del volumen de sólidos y disminución de la distancia entre las partículas, es el período en que el efecto filler es más importante cuando el C-S-H precipitado une las partículas (BERODIER; SCRIVENER, 2014).

Con respecto al período de desaceleración, la inclusión de MK, adelanta su inicio y disminuye su duración (Tabla 7). En esta etapa se forma la etringita y el monosulfoaluminato.

\section{Análisis termogravimétrico}

Las Figuras 5a y 5b muestran respectivamente la evolución de la cantidad de agua combinada (correspondiente a los compuestos hidratados formados de los aluminatos, silicatos, sulfatos y de magnesio) y portlandita. Los resultados están normalizados en relación a la cantidad de cemento. En la Tabla 8 se muestran los valores numéricos de la cantidad de portlandita.

Se observa que la cantidad de agua combinada (Figura 5a) aumenta sensiblemente hasta las 24 horas, para enseguida casi estabilizar, lo mismo puede ser dicho para la portlandita (Figura 5b). Las alteraciones observadas durante este período pueden ser atribuidas a variaciones de ensayo. La sustitución de cemento por MK manteniendo la relación agua/sólido, significa mayor disponibilidad de agua para la hidratación de la fracción clinker, aumentando la cantidad de agua combinada, contribuyendo también al aumento de productos hidratados. En este período aún no se tiene reacción puzolánica, con consumo de portlandita.

Como se observa en la Tabla 8, a partir de las 24 horas, existe una disminución en el contenido de portlandita, pero tal disminución es mínima, para ambas sustituciones de MK. Por lo que, hasta las 72 horas de análisis el MK actúa predominantemente en forma física y su efecto puzolánico se presentará en edades avanzadas. En el estudio de Kocaba, Gallucci y Scrivener (2012), para distinguir el efecto físico, de la reactividad puzolánica de un SCM, utilizan como sustitución un material inerte y de similar diámetro (normalmente cuarzo). Comparando estas dos curvas, una con un material inerte y la otra con material puzolánico, se puede distinguir ambos efectos.

\section{Difracción de rayos $\mathrm{X}$}

La Figura 6 muestra una comparación de los difractogramas de rayos $\mathrm{X}$, entre las pastas de referencia y con $20 \%$ de sustitución de MK, para edades de 2, 8, 12 y 24 horas de hidratación. En los diagramas, a las 24 horas de hidratación se aprecia fases remanecientes del clinker, como el $\mathrm{C}_{3} \mathrm{~S}$. Comparando los difractogramas se observa que la sustitución de MK favorece la disolución del $\mathrm{C}_{3} \mathrm{~S}$, lo cual se verifica por la disminución en la intensidad de los picos localizados en $29,5^{\circ}, 30^{\circ}$, $34,2^{\circ}$ y $38,7^{\circ} 2 \theta$. Tal disminución está directamente relacionada con la formación de productos hidratados como: la portlandita, con aumento de intensidad en los picos localizados en $18^{\circ}$ y $34^{\circ} 2 \theta$ y el C-S-H, con pico localizado en $2,6^{\circ} 2 \theta$. Esto indica que la presencia de MK acelera las reacciones de hidratación durante las 24 horas de estudio.

Por otra parte, en las pastas con sustitución de MK se mantiene el pico correspondiente al cuarzo, localizados en $26,7^{\circ} 2 \theta$, oriundo del $\mathrm{MK}$. Su intensidad se mantiene constante por tratarse de un mineral inerte no reactivo. El pico referente a la portlandita disminuye en este periodo por adsorción de los iones $\mathrm{Ca}^{2+}$ en las partículas del MK.

\section{Retracción química}

Las Figuras 7a y 7b muestran respectivamente las curvas de retracción química y tasa de retracción química, para la pasta de referencia y sustituciones de 5, 10 y 20\% de MK. Las curvas están normalizadas con respecto a la cantidad de cemento. 
La curva de retracción química aumenta conforme se adiciona mayor cantidad de MK. La Tabla 9 muestra los valores obtenidos, a las 72 horas de hidratación, la retracción química aumenta de 0,0438 a $0,5049 \mathrm{~mL} / \mathrm{g}(25,3 \%$ de incremento) para la pasta de referencia y la de mayor sustitución, respectivamente. Esto es debido a que la presencia de MK acelera la hidratación por efecto de nucleación del C-S-H, lo cual también fue observado por la calorimetría y termogravimetría descritos anteriormente. Como se sabe, la retracción química está directamente relacionada con la formación de C-S-H, por lo tanto la presencia de MK favorece su formación, pero incrementa la retracción química.
La Figura 7b muestra la tasa de retracción química (primera derivada de la curva de retracción química). En esta curva también es posible observar los eventos que ocurren durante el desarrollo de la cinética de hidratación. Con el aumento gradual de MK se presentan modificaciones en la curva de tasa de retracción química. La duración del período de aceleración disminuye, existe una diferencia de 3 horas entre la pasta de referencia y la de mayor sustitución. Además, la intensidad del pico máximo aumenta. Berodier (2015) menciona que para una edad de hidratación menor a 10 horas, el C-S-H llena los espacios exteriores disponibles, mientras que para edades mayores, el C-S-H empieza a llenar los poros capilares (espacios internos y externos).

Tabla 7 - Tiempo de duración y flujo de calor durante el período de desaceleración

\begin{tabular}{c|c|c|c|c}
\hline \multicolumn{5}{c}{ Período de desaceleración } \\
\hline Muestra & Inicio (h) & Final (h) & $\begin{array}{c}\text { Tiempo } \\
\text { duración (h) }\end{array}$ & $\begin{array}{c}\text { Flujo de calor } \\
\text { (mW/g) }\end{array}$ \\
\hline CPV 0\%MK & 11,0 & 18,0 & 7,0 & 2,01 \\
CPV 5\%MK & 10,0 & 16,0 & 6,0 & 2,34 \\
CPV 10\%MK & 9,8 & 14,0 & 4,2 & 2,86 \\
CPV 20\%MK & 8,0 & 12,0 & 4,0 & 3,51 \\
\hline
\end{tabular}

Figura 5 - Evolución de (a) agua combinada y (b) portlandita - para la pasta de referencia y sustituciones de 10 y $20 \%$ de MK
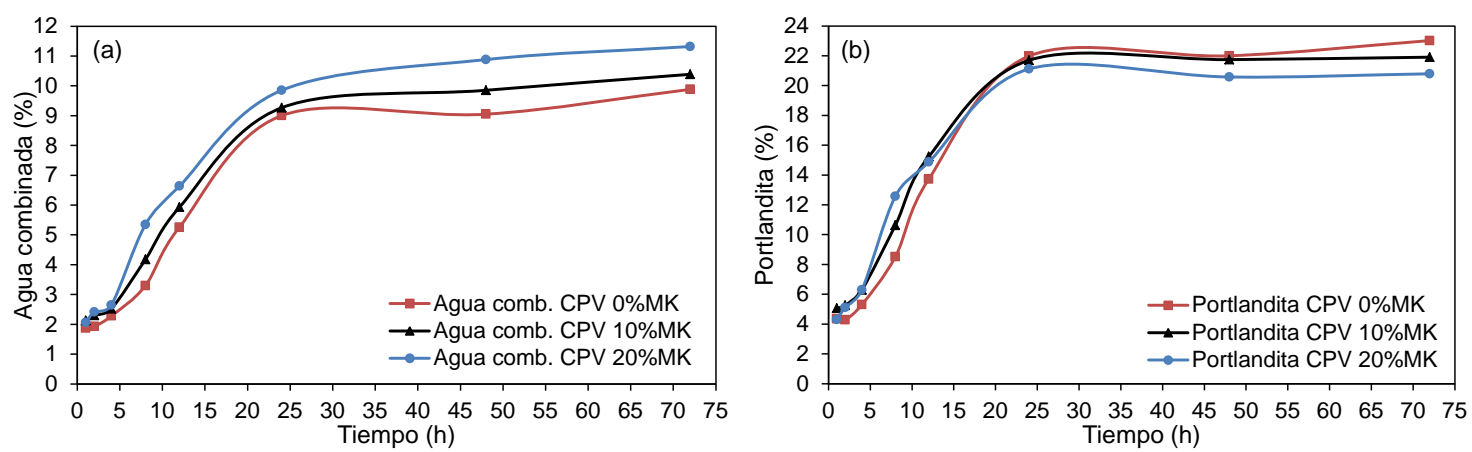

Tabla 8 - Valores de portlandita hasta 72 horas de hidratación

\begin{tabular}{|c|c|c|c|c|c|}
\hline Tiempo & \multicolumn{5}{|c|}{ Muestra } \\
\hline (h) & CPV 0\%МK & CPV 10\%MK & CPV 20\%MK & $\Delta 10 \% \mathrm{MK}^{*}$ & $\Delta 20 \% \mathrm{MK}^{* *}$ \\
\hline 1 & 4,4 & 5,1 & 4,4 & 0,7 & 0,0 \\
\hline 2 & 4,3 & 5,3 & 5,1 & 1,0 & 0,8 \\
\hline 4 & 5,3 & 6,3 & 6,3 & 1,0 & 1,0 \\
\hline 8 & 8,5 & 10,6 & 12,6 & 2,1 & 4,1 \\
\hline 12 & 13,7 & 15,2 & 14,9 & 1,5 & 1,2 \\
\hline 24 & 22,0 & 21,7 & 21,1 & $-0,3$ & $-0,9$ \\
\hline 48 & 22,0 & 21,7 & 20,6 & $-0,3$ & $-1,4$ \\
\hline 72 & 23,0 & 21,9 & 20,8 & $-1,1$ & $-2,2$ \\
\hline
\end{tabular}

Nota: $* \Delta 10 \%$ MK: CPV10\%MK - CPV; y ** $\Delta 20 \%$ MK: CPV20\%MK - CPV. 
Figura 6 - Comparación de difractogramas de rayos $X$ con edades de hidratación de 2, 8, 12 y 24 horas, para la pasta de referencia y con $20 \%$ de MK
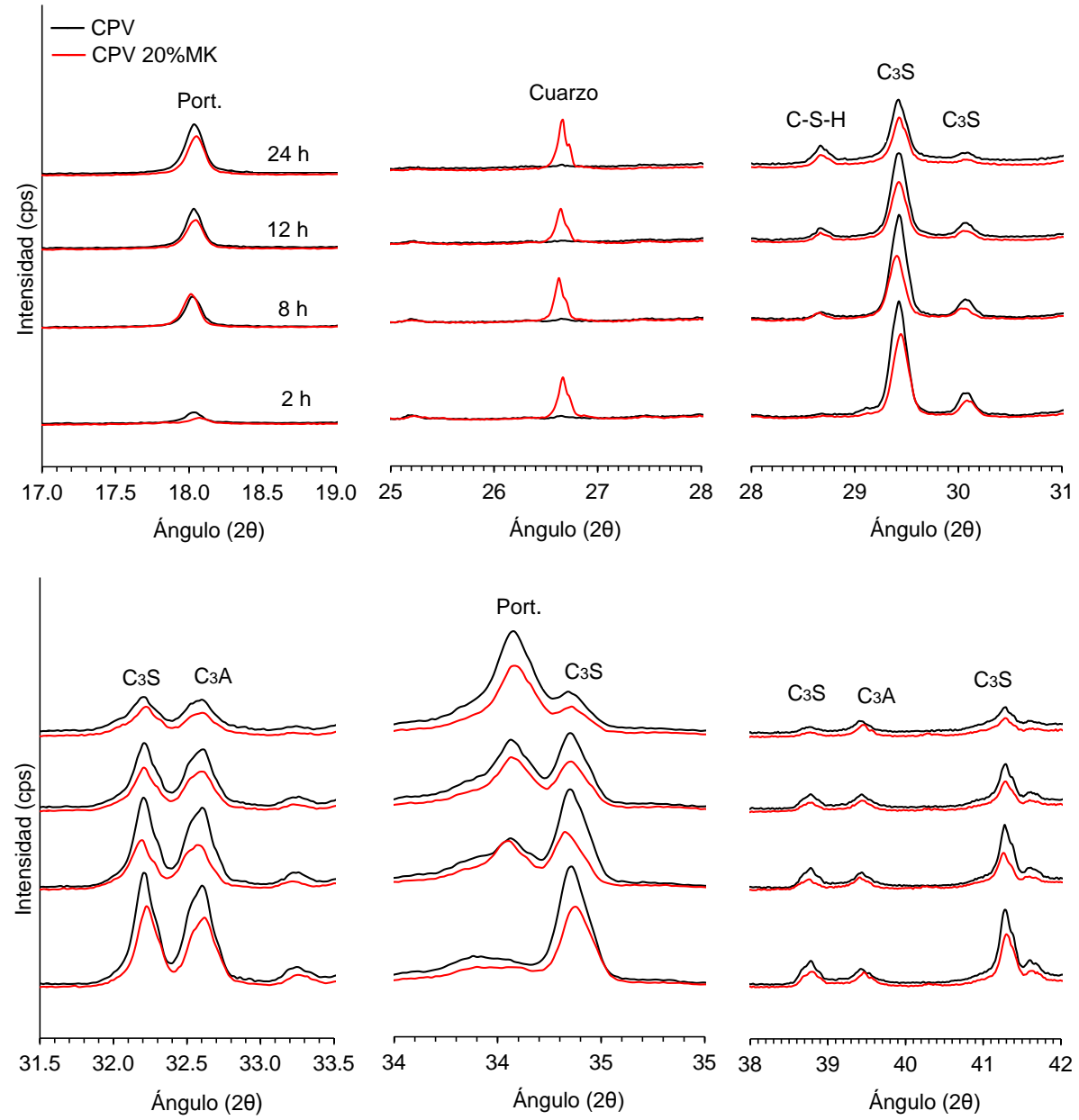

Figura 7 - (a) Retracción química y (b) Tasa de retracción química - para la pasta de referencia y sustituciones de 5,10 y $20 \%$ de MK
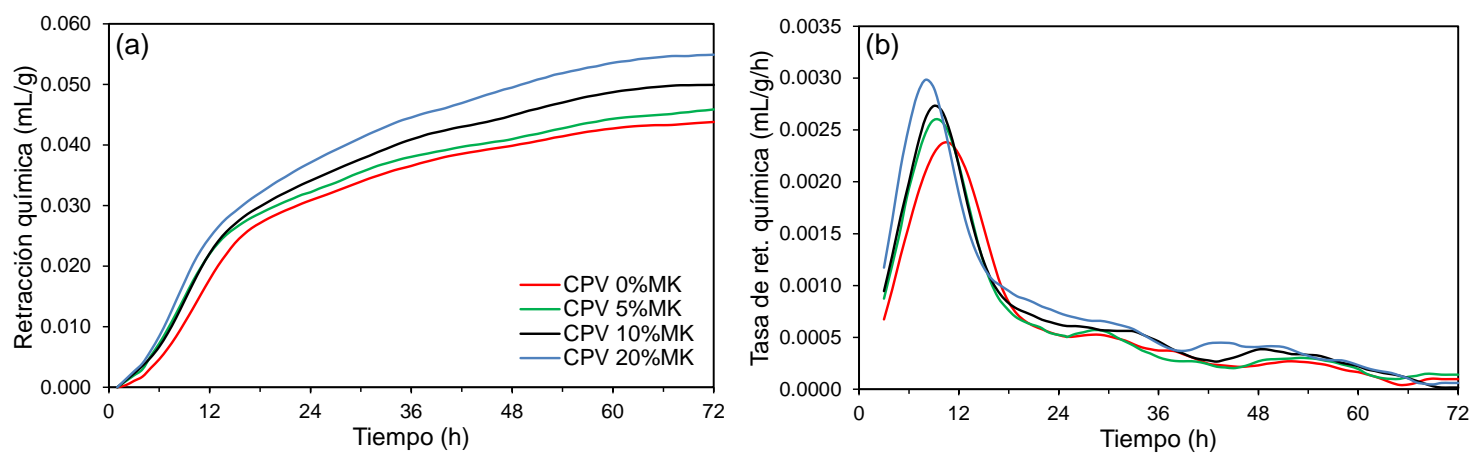

Tabla 9 - Resultados de retracción química para sustituciones de 5, 10 y $20 \%$ de MK

\begin{tabular}{c|c|c|c|c}
\hline \multirow{2}{*}{ Muestra } & \multicolumn{4}{|c}{ Retracción química (mL/g) } \\
\cline { 2 - 5 } & $\mathbf{1 2} \mathbf{~ h}$ & $\mathbf{2 4} \mathbf{h}$ & $\mathbf{4 8 ~ h}$ & $\mathbf{7 2 ~ h}$ \\
\hline CPV 0\%MK & $0,0178(-)$ & $0,0309(-)$ & $0,0399(-)$ & $0,0438(-)$ \\
CPV 5\%MK & $0,0220(+23,6 \%)$ & $0,0322(+4,2 \%)$ & $0,0410(+2,8 \%)$ & $0,0459(+4,8 \%)$ \\
CPV 10\%MK & $0,0221(+24,2 \%)$ & $0,0341(+10,4 \%)$ & $0,0449(+12,5 \%)$ & $0,0499(+13,9 \%)$ \\
CPV 20\%MK & $0,0247(+38,8 \%)$ & $0,0371(+20,1 \%)$ & $0,0495(+24,1 \%)$ & $0,0549(+25,3 \%)$ \\
\hline
\end{tabular}


Las oscilaciones en los resultados que se muestran en edades avanzadas, pueden ser explicadas por efectos de dilatación del agua. Es por ello que, para minimizar el efecto de la temperatura en los resultados, es recomendable utilizar un baño térmico de precisión y realizar el ensayo en una sala con temperatura controlada.

\section{Comparación de resultados entre retracción química y calorimetría}

La Figura 8 muestra comparaciones entre las curvas de flujo de calor y tasa de retracción química, para la pasta de referencia y sustituciones de 5, 10 y 20\% de metacaolín.

En las Figuras 8a, 8b, 8c y 8d, se observan que las curvas de flujo de calor y tasa de retracción química tienen forma similar, para todas las sustituciones usadas. Además, es posible distinguir las etapas de la cinética de hidratación mediante ambos métodos. Estudios como el de Parrot et al. (1990) señalan que existe una relación lineal entre la retracción química y el calor de hidratación. Igualmente, Lura,
Winnefeld y Klemm (2010) muestran que ambos resultados son comparables bajo las mismas condiciones de hidratación. Por lo tanto, en virtud al incremento y diversidad de SCM el uso de ambos métodos, retracción química y calorimetría, son cada vez más frecuentes en el estudio de la cinética de hidratación.

La Tabla 10 presenta una comparación del tiempo de ocurrencia del pico de la etapa de aceleración, determinado por calorimetría y retracción química. En general todas las muestras presentan resultados iguales, solo con diferencias de 0,5 y 0,25 horas, para 5 y $10 \%$ de sustitución, respectivamente. Por tanto, mediante la retracción química también es posible obtener este parámetro.

En la Figura 9 se presentan correlaciones entre los resultados de retracción química y calor acumulado. Para todas las sustituciones estudiadas se observa una relación lineal, con coeficientes de determinación $\mathrm{R}^{2}=0,99$. Resultados similares fueron encontrados por Lura, Winnefeld y Klemm (2010).

Figura 8 - Comparación entre las curvas de flujo de calor y tasa de retracción química para: (a) CPV 0\%MK, (b) CPV 5\%MK, (c) CPV 10\%MK y (d) CPV 20\%MK
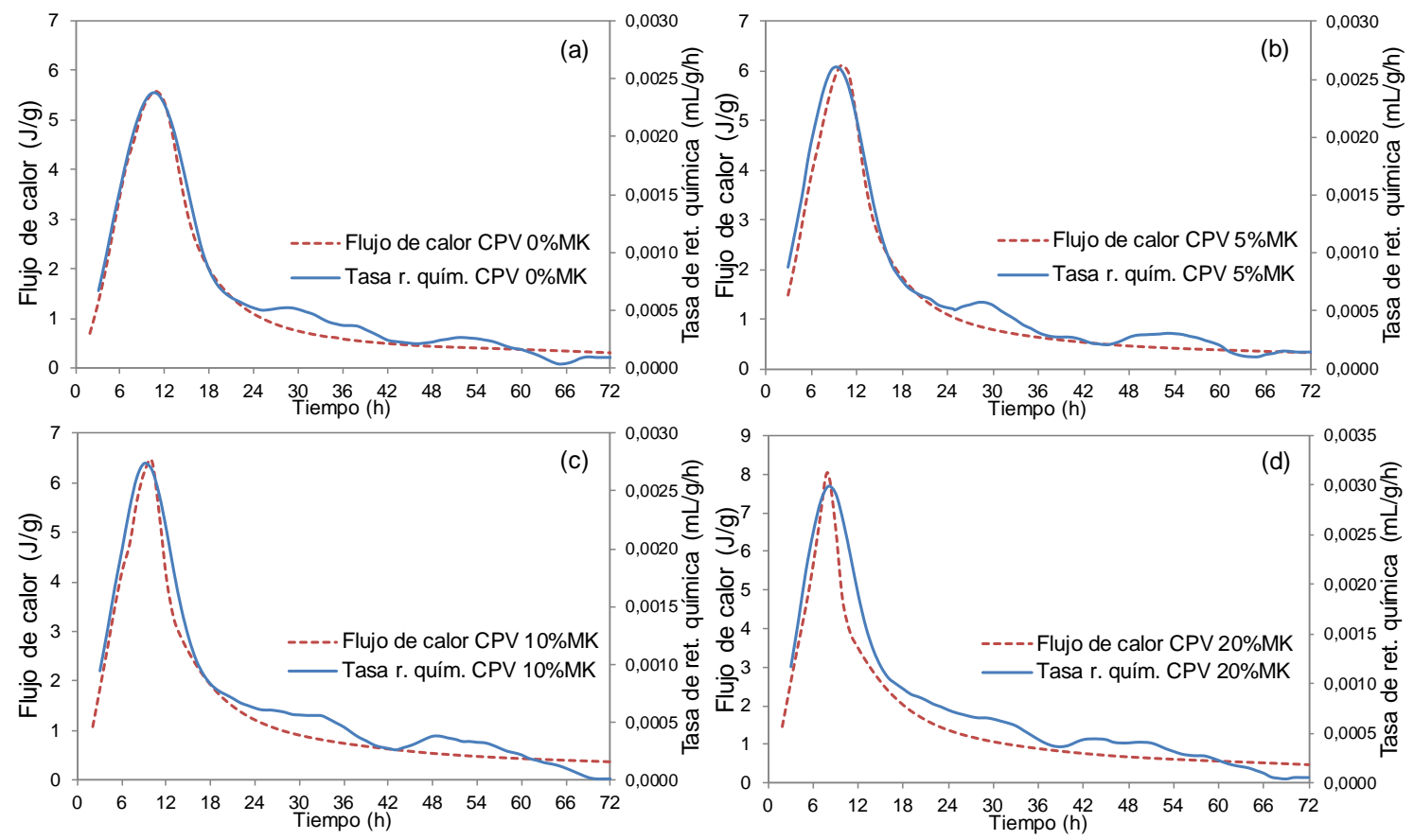

Tabla 10 - Comparación del tiempo en que ocurre el pico de aceleración mediante los dos métodos

\begin{tabular}{l|c|c|c}
\hline \multirow{2}{*}{ Material } & \multicolumn{2}{|c|}{ Tiempo del pico de aceleración (horas) } & $\begin{array}{c}\text { Diferencia } \\
\text { (horas) }\end{array}$ \\
\cline { 2 - 4 } & Calorimetría & Retracción química & 0,00 \\
CPV 0\%MK & 11,00 & 11,00 & 0,50 \\
CPV 5\%MK & 10,00 & 9,50 & 0,25 \\
CPV 10\%MK & 9,75 & 9,50 & 0,00 \\
CPV 20\%MK & 8,00 & 8,00 & \\
\hline
\end{tabular}


Figura 9 - Correlaciones entre retracción química y calor acumulado para (a) pasta de referencia y sustituciones de (b) $5 \%$, (c) $10 \%$ y (d) $20 \%$ de MK
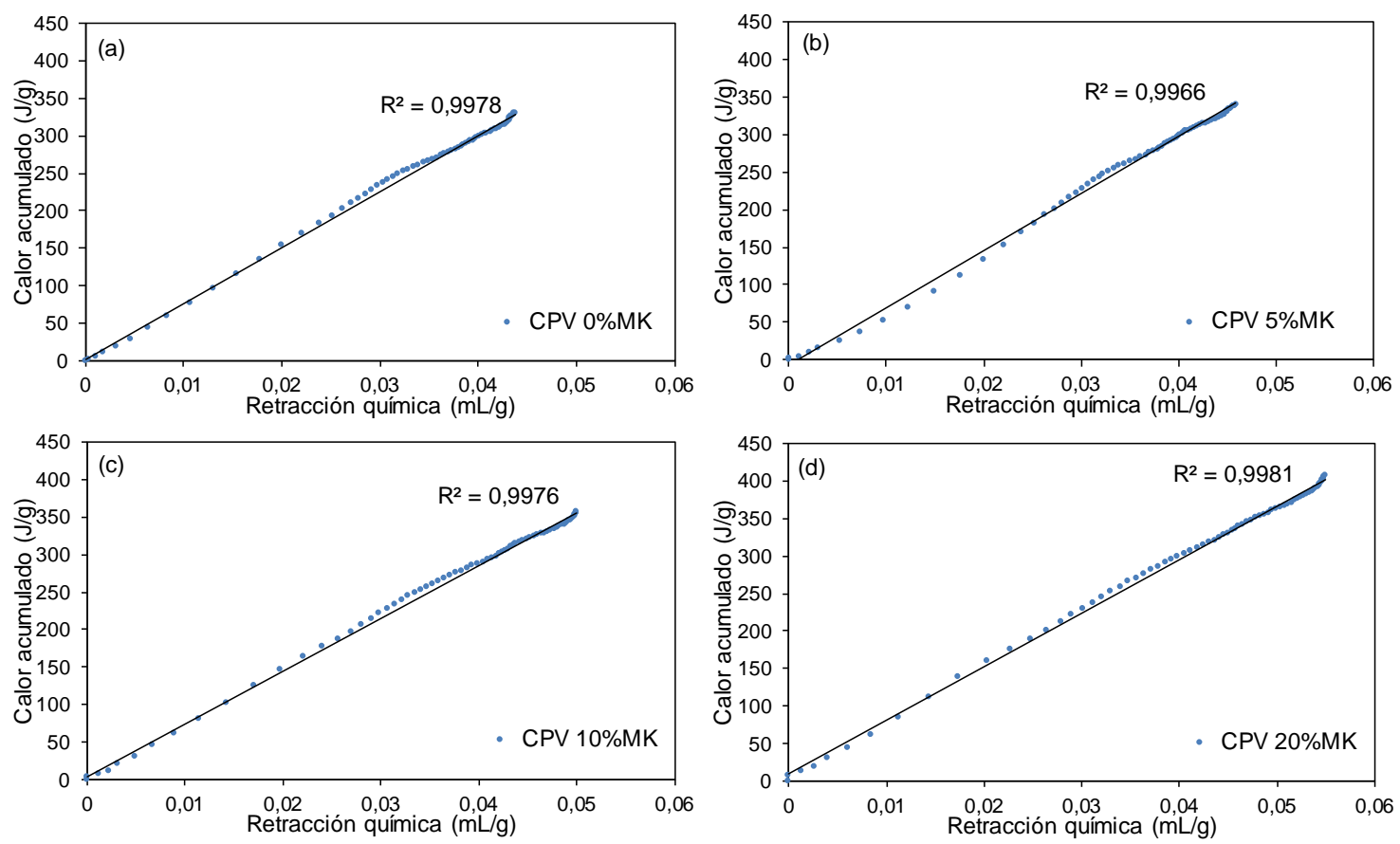

En la Figura 10 y conforme los datos de las Tablas 5 y 9, se muestra una comparación entre el incremento del calor acumulado y de la retracción química, para sustituciones de 5, 10 y 20 \% de MK. En ambas se observa que durante las primeras 24 horas de hidratación ocurre un incremento considerable, siendo más notorio para la mayor sustitución, luego de esta etapa el incremento empieza a estabilizarse.

\section{Conclusiones}

A partir de los resultados de la retracción química, también es posible obtener información de la cinética de hidratación. Al comparar los resultados de cinética de hidratación, obtenidos tanto por retracción química como por calorimetría, se comprobó que tienen una relación excelente. Por tanto, los resultados de los ensayos de retracción química permiten estudiar la cinética de hidratación de cementos, sin que sea necesario adquirir dispendiosos calorímetros isotérmicos.

La presencia de MK en pastas cementicias genera reacciones adicionales de hidratación, sobre todo mayor cantidad de C-S-H. Esto es debido a su presencia física, siendo generador de puntos extras de nucleación, favoreciendo la precipitación del CS-H y de la portlandita; además de la disminución de la distancia entre sus partículas. Esto hace que

exista, por un lado disminución de volumen (retracción química) y por otro, reacciones exotérmicas adicionales (captadas por el calorímetro). Entonces, tanto la retracción química como el calor acumulado, aumentan con la sustitución gradual de MK.

Las curvas de flujo de calor y tasa de retracción química se ven afectadas con la adición progresiva de MK, estos cambios son:

(a) la duración del período de aceleración disminuye, existe una diferencia de 3 horas entre la pasta de referencia y la de mayor sustitución; y

(b) la intensidad del pico de aceleración aumenta, generando mayor flujo de calor liberado y tasa de retracción química, respectivamente.

Estos cambios indican que la inclusión de MK acelera las reacciones de hidratación.

El análisis termogravimétrico confirma los resultados obtenidos por calorimetría y retracción química. La adición progresiva de MK actúa en dos etapas, primero acelera las reacciones de hidratación, principalmente debido a su efecto físico y en una segunda etapa a partir de 24 horas, empieza a participar de las reacciones de hidratación, disminuyendo la cantidad de portlandita. 
Figura 10 - Comparación entre los incrementos de (a) calor acumulado y (b) retracción química

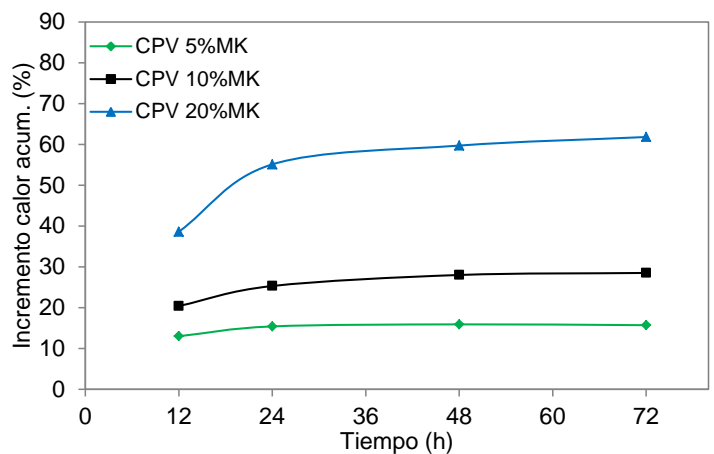

(a)

Los diagramas de difracción de rayos X hasta las 24 horas de análisis muestran que la inclusión de MK favorece la disolución del $\mathrm{C}_{3} \mathrm{~S}$, la precipitación del C-S-H y portlandita. En las pastas con inclusión de MK se observa que se mantiene el pico del cuarzo oriundo del MK, durante todas las edades de hidratación estudiadas.

\section{Referencias}

AMBROISE, J.; MAXIMILIEN, S.; PERA, J. Properties of Metakaolin Blended Cements.

Advanced Cement Based Materials, v. 1, n. 4, p. 161-168, 1994.

ANTONI, M. et al. Cement Substitution by a Combination of Metakaolin and Limestone. Cement and Concrete Research, v. 42, n. 12, p. 1579-1589, dez. 2012.

\section{AMERICAN SOCIETY FOR TESTING AND} MATERIALS. C-1608: standard test method for chemical shrinkage of hydraulic cement paste. West Conshohocken, 2012.

\section{AMERICAN SOCIETY FOR TESTING AND} MATERIALS. C-1679: standard practice for measuring hydration kinetics of hydraulic cementitious mixtures using isothermal calorimetry.West Conshohocken, 2014

\section{BERODIER, E. Impact of the Suplementary} Cementitious Materials on the Kinetics and Micoestruture Development of Cement Hydration. Suiza, 2015. PhD Thesis - École Polytechnique Fédérale de Lausanne, Suiza, 2015.

BERODIER, E.; SCRIVENER, K. Understanding the Filler Effect on the Nucleation and Growth of C-S-H. Journal of the American Ceramic Society, v. 97, n. 12, p. 3764-3773, dez. 2014.

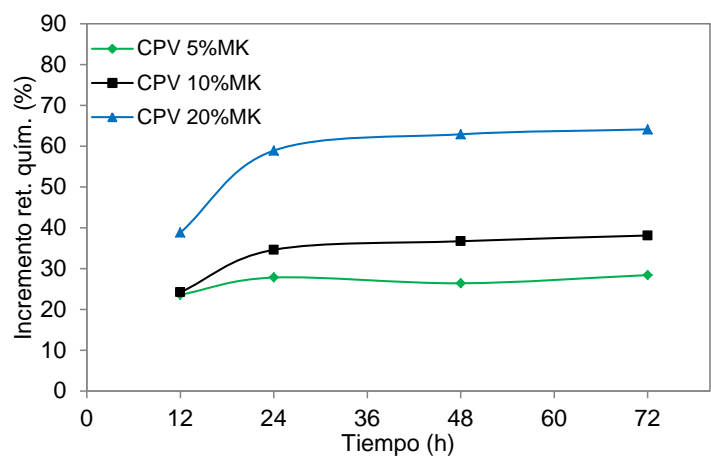

(b)

ESTRADA, D. P. Influencia del Metacaolin en las Propiedades en Estado Fresco del Cemento Portland: hidratación y retracción química. Dissertação (Mestrado em Engenharia Civil) Escola Politecnica, Universidade de São Paulo, São Paulo, 2016.

ESTRADA, D. P.; JOHN, V. M.; CINCOTTO, M. A. Métodos de Determinación de la Cinética de Hidratación Mediante la Retracción Química. Ambiente Construido, v. 17, n. 4, p. 109-124, out./dez. 2017.

JUENGER, M. C. G.; SIDDIQUE, R. Recent Advances in Understanding the Role of Supplementary Cementitious Materials in Concrete. Cement and Concrete Research, v. 78, Part A, p. 71-80, dez. 2015.

KOCABA, V.; GALLUCCI, E.; SCRIVENER, K. L. Methods for Determination of Degree of Reaction of Slag in Blended Cement Pastes. Cement and Concrete Research, v. 42, n. 3, p. 511-525, mar. 2012.

LAGIER, F.; KURTIS, K. E. Influence of Portland Cement Composition on Early Age Reactions With Metakaolin. Cement and Concrete Research, v. 37, n. 10, p. 1411-1417, out. 2007.

LOTHENBACH, B.; SCRIVENER, K.; HOOTON, R. D. Supplementary Cementitious Materials. Cement and Concrete Research, v. 41, n. 12, p. 1244-1256, dez. 2011.

LURA, P.; WINNEFELD, F.; KLEMM, S. Simultaneous Measurements of Heat of Hydration and Chemical Shrinkage on Hardening Cement Pastes. Journal of Thermal Analysis and Calorimetry, v. 101, n. 3, p. 925-932, 2010.

PARROTT, L. J. et al. Monitoring Portland Cement Hydration: comparison of methods. Cement and Concrete Research, v. 20, n. 6, p. 919-926, nov. 1990. 
POON, C.-S. et al. Rate of Pozzolanic Reaction of Metakaolin in High-Performance Cement Pastes. Cement and Concrete Research, v. 31, n. 9, p. 1301-1306, set. 2001.

RASHAD, A. M. Metakaolin as Cementitious Material: history, scours, production and composition: a comprehensive overview.

Construction and Building Materials, v. 41, p. 303-318, 2013.

SABIR, B. B.; WILD, S.; BAI, J. Metakaolin and Calcined Clays as Pozzolans For Concrete: a review. Cement and Concrete Composites, v. 23, n. 6, p. 441-454, dez. 2001.

SCRIVENER, K. L.; JUILLAND, P.; MONTEIRO, P. J. M. Advances in Understanding Hydration of Portland Cement. Cement and Concrete Research, v. 78, Part A, p. 38-56, dez. 2015.
SIDDIQUE, R.; KLAUS, J. Influence of Metakaolin on the Properties of Mortar and Concrete: a review. Applied Clay Science, v. 43, n. 3/4, p. 392-400, mar. 2009.

VANCE, K. et al. Hydration and Strength Development in Ternary Portland Cement Blends Containing Limestone and Fly Ash or Metakaolin. Cement and Concrete Composites, v. 39, p. 93103, maio 2013.

\section{Agradecimentos}

Los autores agradecen a CAPES por el soporte económico para la realización de este trabajo.

\section{Delver Plinio Estrada Caceres}

Departamento de Engenharia de Construção Civil, Escola Politécnica | Universidade de São Paulo | Av. Professor Almeida Prado, Trav. 2, 87, Cidade Universitária | São Paulo - SP - Brasil | CEP 05508-900 | Tel.: (11) 3091-5459 | E-mail: delver.estrada@alumni.usp.br

\section{Roberto Cesar de Oliveira Romano}

Escola Politécnica | Universidade de São Paulo | Tel.: (11) 3091-5382 | E-mail: rcorjau@gmail.com

\section{Vanderley Moacyr J ohn}

Departamento de Engenharia de Construção Civil, Escola Politécnica | Universidade de São Paulo | Tel.: (11) 3091-5794 |

E-mail: vmjohn@lme.pcc.usp.br

\section{Maria Alba Cincotto}

Departamento de Engenharia de Construção Civil, Escola Politécnica | Universidade de São Paulo | Tel.: (11) 3091-5792 | E-mail: cincotto@usp.br

\section{Revista Ambiente Construído}

Associação Nacional de Tecnologia do Ambiente Construído

Av. Osvaldo Aranha, 99 - 3o andar, Centro

$$
\begin{gathered}
\text { Porto Alegre - RS - Brasil } \\
\text { CEP 90035-190 }
\end{gathered}
$$

Telefone: +55 (51) 3308-4084

Fax: +55 (51) 3308-4054

www. seer. ufrgs. br/ ambienteconstruido

E-mail: ambienteconstruido@ufrgs.br 